\title{
Extracting hidden-photon dark matter from an LC-circuit
}

\author{
Paola Arias ${ }^{1, a}$, Ariel Arza ${ }^{1}$, Babette Döbrich ${ }^{2,3}$, Jorge Gamboa ${ }^{1}$, Fernando Méndez ${ }^{1}$ \\ ${ }^{1}$ Departmento de Física, Universidad de Santiago de Chile, Casilla 307, Santiago, Chile \\ ${ }^{2}$ Deutsches Elektronen-Synchrotron DESY, Notkestr. 85, 22607 Hamburg, Germany \\ ${ }^{3}$ Present address: CERN, 1211 Geneva 23, Switzerland
}

Received: 15 April 2015 / Accepted: 23 June 2015 / Published online: 4 July 2015

(C) The Author(s) 2015. This article is published with open access at Springerlink.com

\begin{abstract}
We point out that a cold dark matter condensate made of gauge bosons from an extra hidden U(1) sectordubbed hidden photons — can create a small, oscillating electric density current. Thus, they could also be searched for in the recently proposed LC-circuit setup conceived for axion cold dark matter search by Sikivie, Sullivan and Tanner. We estimate the sensitivity of this setup for hidden-photon cold dark matter and we find it could cover a sizable, so far unexplored parameter space.
\end{abstract}

\section{Introduction}

Nowadays, direct dark matter searches are mainly taking two alternative and complementary routes: one of them aims to detect high-mass candidates-so-called Weakly Interacting Massive Particles (WIMPs) — exploiting scattering experiments [1], and the other one looks for light mass candidatesso-called Weakly Interacting Slim Particles (WISPs)—using precision experiments and strong magnetic fields [2].

Among WISPs, the axion is a prime candidate. It was originally proposed as a mechanism to solve the strong $\mathrm{CP}$ problem [3-5]. Soon after this proposal, it was realized that axions can be non-thermally produced by a misalignment mechanism, making it a strong cold dark matter (CDM) candidate in the range of masses $m_{a} \lesssim 10^{-4} \mathrm{eV}$ [6-8].

A common feature among WISPs is their weak coupling to the Standard Model, and the smallness of their masses. This is often a heritage from the high-energy scale at which their underlying symmetries break. Many indirect astrophysical observations have placed strong constraints on these particles [9], but there is still plenty of parameter space in which they could hide. In particular, the parameter space where they can be CDM remains still quite open.

The WISPs relevant to this study are hidden sector $U(1)$ gauge bosons [10], also known as paraphotons, or hidden

a e-mail: paola.arias.r@usach.cl photons. Remarkably, the same non-thermal mechanism of axion CDM production also works to produce a condensate of cold hidden photons $[11,12]$, whose viable parameter space spans a wide range and remains almost unconstrained by observations.

Consequently, experimental efforts have increased in the last years, and several precision experiments have been and will be set up, like ADMX [13], ALPS [14,15], CAST, CROWS [16], IAXO [17] (just to name a few) and help to cover some of the unexplored parameter space.

Novel proposals, specially thought to reach the hinted cold dark matter parameter space have emerged, such as a dish antenna experiment $[18,19]$. In this study we want to revisit the proposal made by Sikivie, Sullivan and Tanner [20], in which they explore the particular form taken by the Maxwell equations if the axion CDM is present.

This new setup has interesting features; the first is the simplicity of the idea, namely an LC-circuit carrying an electric current generated by CDM axions in an external magnetic field. Secondly, the signal produced by axions can be amplified by the circuit, making it detectable by magnetic flux detection techniques.

The aim of this letter is to show that hidden-photon CDM can also provide an oscillating electric current, without the need of an external electromagnetic field, which can act as a source for the proposed experiment [20]. Therefore, this setup can also hunt for these particles. We note that LC-circuits have been mentioned in [21] as hidden-photon receivers, however, not adapted to the context of dark matter detection.

The paper is organized as follows: in Sect. 2 we briefly review the operating mechanism of the LC circuit designed to detect axions. In Sect. 3 we show how an oscillating current from hidden-photon CDM emerges from the coupling of the latter with photons, and we obtain the sensitivity of the experiment proposed in [20] for hidden photons. Finally in Sect. 4 we conclude. 


\section{Essentials of the axion search with an LC-circuit}

Let us recall the essentials of the proposal made in [20]. The idea exploits the fact that the coupling of axions and photons

$\mathcal{L}=-g a F_{\mu \nu} \tilde{F}^{\mu \nu}$,

gives rise to a modified electrodynamics

$\nabla \times \mathbf{B}-\frac{\partial \mathbf{E}}{\partial t}=-g \mathbf{B} \frac{\mathrm{d} a}{\mathrm{~d} t}+\mathbf{J}_{\mathrm{ext}}$

where $g$ is the coupling constant between axions and photons, $\mathbf{J}_{\text {ext }}$ is an external source and a homogeneous axion field is assumed i.e. $a=a(t)$ and therefore spatial derivatives of this field are neglected. This is approximately valid for axion DM.

Note that Eq. (2) contains the effective displacement current $\mathbf{j}_{\mathbf{a}}=-g \dot{a} \mathbf{B}_{\mathbf{0}}$ which emerges when an external magnetic field $\mathbf{B}_{0}$ is turned on and, as a consequence, the current $\mathbf{j}_{a}$ becomes a source for a magnetic field $\mathbf{B}_{a}$ through the equation $\nabla \times \mathbf{B}_{a}=\mathbf{j}_{a}$.

Thus, the idea is to insert part of an LC-circuit into a region with an external magnetic field. Figure 1 mimics the setup of [20], where the external magnetic field $\mathbf{B}_{\mathbf{0}}$ around the passive part of the circuit is omitted for a hidden-photon search (see below).

The modification (2) implies that for the setup [20] in the presence of axion CDM an electric current will flow through the LC-circuit, eventually in the resonance regime for $1 / \sqrt{L C} \approx m_{a}$.

The important thing to note, however, is the following: once the electric current is produced in the circuit, the magnetic flux in the coil measured by a magnetometer, here a SQUID, is related to the dark matter axion properties.

Indeed, the amplitude of the magnetic field $B_{d}$ in the coil that will be detected by the SQUID is given by

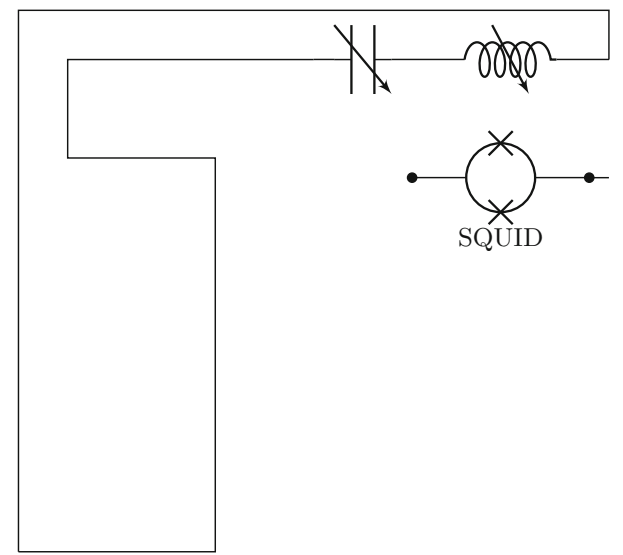

Fig. 1 Sketch of the experiment. In contrast to the original SikivieSullivan-Tanner setup [20], no external magnet is needed. As the hidden-photon DM might have a net polarization, orthogonal circuits could be foreseen to minimize the effect of $\kappa$ (see text for details)
$B_{d} \simeq \frac{N_{d} Q}{2 r_{d} L} V_{m} g B_{0} \sqrt{2 \rho_{\mathrm{DM}}}$

where $N_{d}, r_{d}, V_{m}$ are parameters of the device keeping the notation of [20]: $N_{d}$ is the number of turns and $r_{d}$ is radius of the small coil on the right hand side of Fig. $1, V_{m}$ is a parameter with dimensions of volume, which appears in the integration of the magnetic flux in the circuit; see below. $L$ is the inductance of the entire system, $Q$ is the quality factor of the circuit, $B_{0}$ is the magnitude of the external magnetic field and $\rho_{\mathrm{DM}}$ is the dark matter energy density of the axion field.

In their proposal [20], the authors have assumed a superconducting circuit, working at a temperature of the order of $T \sim 0.5 \mathrm{mK}$, a SQUID sensitivity of the order of $10^{-15} \mathrm{~T}$, a quality factor of the circuit $Q=10^{4}$, and two possible magnets: the ADMX magnet or CMS magnet (see details in [20]).

\section{Connection with hidden photons}

In this section we will argue that an oscillating current also emerges if the CDM content is composed of hidden photons.

In the hidden-photon model we are interested in, the dominant interaction between hidden photons and our visible sector is via a kinetic mixing term. At low energies the effective Lagrangian is given by

$\mathcal{L}=-\frac{1}{4} F_{\mu \nu} F^{\mu \nu}-\frac{1}{4} X_{\mu \nu} X^{\mu \nu}-\frac{\chi}{2} F_{\mu \nu} X^{\mu \nu}+\frac{m_{\gamma^{\prime}}^{2}}{2} X_{\mu} X^{\mu}$.

Here $F_{\mu \nu}$ is the field strength associated with photons $\left(A_{\mu}\right)$ and $X_{\mu v}$ the analog for hidden photons $\left(X_{\mu}\right)$. The $\chi$ parametrizes the strength of the coupling between both, and is predicted to be small [22-25]. The mass of the hidden photon $m_{\gamma^{\prime}}$ can be generated via a hidden-Higgs mechanism, or a Stückelberg mechanism.

In an analog way to axions, hidden-photon CDM can be considered as a homogeneous field in space, given by $\mathbf{X}(t)=\mathbf{X}_{\mathrm{DM}} \mathrm{e}^{-i m_{\gamma^{\prime}} t}$, where $\mathbf{X}_{\mathrm{DM}}$ is the DM vector, and due to its vector nature, a cold condensate of hidden photons can introduce a preferred direction in space.

Effectively a tiny fraction of its energy is invested in an ordinary oscillatory electric field $[18,19]$, given by

$\mathbf{E}_{\mathrm{DM}}=\chi m_{\gamma^{\prime}} \mathbf{X}_{\mathrm{DM}} \mathrm{e}^{-i m_{\gamma^{\prime}} t}$.

Such an electric field will create a displacement current, oscillating at the same frequency as the electric field, $v=$ $0.24 \mathrm{GHz}\left(m_{\gamma^{\prime}} / \mu \mathrm{eV}\right)[18,19]$, given by

$\mathbf{J}_{\mathrm{HP}}=-\frac{\partial \mathbf{E}_{\mathrm{DM}}}{\partial t}$. 
The amplitude, analogously to axions, is related to the CDM local density by noting that the stored energy in the condensate is

$\rho_{\mathrm{DM}} \sim 300 \frac{\mathrm{MeV}}{\mathrm{cm}^{3}}=\frac{m_{\gamma^{\prime}}^{2}}{2}\left\langle\left|\mathbf{X}_{\mathrm{DM}}\right|^{2}\right\rangle$.

Thus, the corresponding magnitude of the current density obtained is

$$
\left|\mathbf{J}_{\mathrm{HP}}\right|=\chi m_{\gamma^{\prime}} \sqrt{2 \rho_{\mathrm{DM}}} .
$$

This current will generate-in principle-oscillating electromagnetic fields. Nevertheless, assuming the experiment is enclosed ${ }^{1}$ in a region of characteristic dimension smaller than $m_{\gamma^{\prime}}^{-1}$, we can work in the magneto-quasistatic limit (also assumed in [20]). Following this approximation, the magnetic field created by the displacement current is just $\nabla \times \mathbf{B}_{\mathrm{HP}}=\mathbf{J}_{\mathrm{HP}}$, and the electric field induced is obtained from $\nabla \times \mathbf{E}_{\mathrm{HP}}=-\partial \mathbf{B}_{\mathrm{HP}} / \partial t$. The latter is suppressed inside the enclosed region in comparison with the magnetic field by $\left|\mathbf{E}_{\mathrm{HP}}\right|=m_{\gamma^{\prime}} r\left|\mathbf{B}_{\mathrm{HP}}\right|$, where $r$ is the radial distance in cylindrical coordinates, with the symmetry axis parallel to the direction of the superconducting wire of Fig. 1. Therefore, in the following, we assume the induced electric field $\left(\mathbf{E}_{\mathrm{HP}}\right)$ does not interfere significantly with the small electronic devices of the circuit.

Note that in the present case of hidden photons, the displacement current, see Eq. (6), has the same direction as the CDM condensate, if effectively it has a preferred direction (see below). In the case of axions, instead, the current density has the direction of the external magnetic field, $\mathbf{B}_{0}$.

The component of the current $\mathbf{J}_{\mathrm{HP}}$ which is parallel to the superconducting wire direction will contribute to create the components of field $\mathbf{B}_{\mathrm{HP}}$ which will be responsible for a non-zero magnetic flux through the same part of the circuit. Namely

$$
\left|\mathbf{J}_{\mathrm{HP} \|}\right|=\left|\mathbf{J}_{\mathrm{HP}} \cos \theta\right|=\left|\mathbf{J}_{\mathrm{HP}}\right| \kappa,
$$

where $\theta$ is the angle between the wire and the direction of the current generated by the hidden-photon condensate, and $\kappa=|\cos \theta|$.

This last analysis yields the two possible scenarios:

(i) The condensate of DM points in a preferred direction in space, $\hat{n}$.

(ii) The condensate of DM is randomly oriented in space.

In the first case each direction $\hat{n}$ is equally probable; a conservative choice for $\kappa$ would be, for instance, that its real value is bigger with a $95 \%$ confidence level, thus $\kappa=0.05$.

\footnotetext{
${ }^{1}$ With enclosed we mean the electric field, $\mathbf{E}_{\mathrm{HP}}$, is set to zero at a boundary.
}

In the second case we consider the average among all possible angles and then $\kappa=0.5$.

On the other hand, if scenario (i) is realized in nature, the lab frame's movement with respect to the rest frame of the DM will likely yield a non-constant $\theta$. In fact, this signal modulation could help to track down the dark matter nature of the signal. Also, additional setups in which an LC loop is oriented orthogonally to the primary setup would allow one to probe the parameter space with $\kappa \simeq 1$.

Assuming the superconducting wire is oriented in the $\hat{z}$ direction, the magnetic field induced by the HP-DM is given by

$\mathbf{B}_{\mathrm{HP}}=-\frac{1}{2} \chi m_{\gamma^{\prime}}^{2}\left|\mathbf{X}_{\mathrm{DM}}\right| \mathrm{e}^{-i m_{\gamma^{\prime}} t} r \kappa \hat{\phi}$.

At this point, let us note that the current generated in the circuit is given by $I=\Phi / L$, where $\Phi$ is the magnetic flux of the field generated by the dark matter, and $L$ is the inductance of the circuit. In the case of axions, the magnetic flux is

$\Phi_{a}=-g B_{0} \dot{a} V_{m}$,

where $V_{m}=\frac{1}{4} l_{m}^{2} r_{m}^{2}$ and $l_{m}$ and $r_{m}$ are the length and width of the circuit loop immersed in the field and thus are limited by the size of the magnet bore.

Since hidden-photon DM does not need an external electromagnetic field to induce a current in the circuit, the latter one is also given by $I=\Phi_{\mathrm{HP}} / L$, and the magnetic flux is

$\Phi_{\mathrm{HP}}=-\kappa \chi m_{\gamma^{\prime}}{ }^{2}\left|\mathbf{X}_{\mathrm{DM}}\right| \mathrm{e}^{-i m_{\gamma^{\prime}} t} V_{m}^{\prime}$,

where $V_{m}^{\prime}$ includes now also the part of the loop not immersed in the external field. We will assume in the following $V_{m}^{\prime} \simeq$ $V_{m}$ to be realized in the setups of [20]. In principle, the additional contribution incorporated in $V_{m}^{\prime}$ can be used to further enhance the signal, however, details depend on the exact geometry of the experimental setup: As $V_{m}, V_{m}^{\prime}$ can be obtained from an integral along the loop-area transversal to $\mathbf{B}_{\mathrm{HP}}$.

As pointed out in [26], the geometry of the outer volume, e.g. a cavity enclosing the LC-circuit, can have positive impact on the overall quality factor. In the following, however, we stick to the original setting of [20].

From the hidden-photon point of view the magnetic flux measured in the coil of the Sikivie et al. setup [20] is given by

$B_{\text {detected }}=\frac{N_{d} Q}{2 r_{d} L} V_{m} \chi m_{\gamma^{\prime}} \sqrt{2 \rho_{\mathrm{DM}}} \kappa$.

To get a sensitivity estimate for hidden photons we consider the isothermal halo model [27], where the local dark matter density is $\rho_{\mathrm{DM}}=0.3 \mathrm{GeV} / \mathrm{cm}^{3}$. The energy dispersion $\delta E \sim 10^{-6} m_{\gamma^{\prime}}$, is then bigger than the one considered 


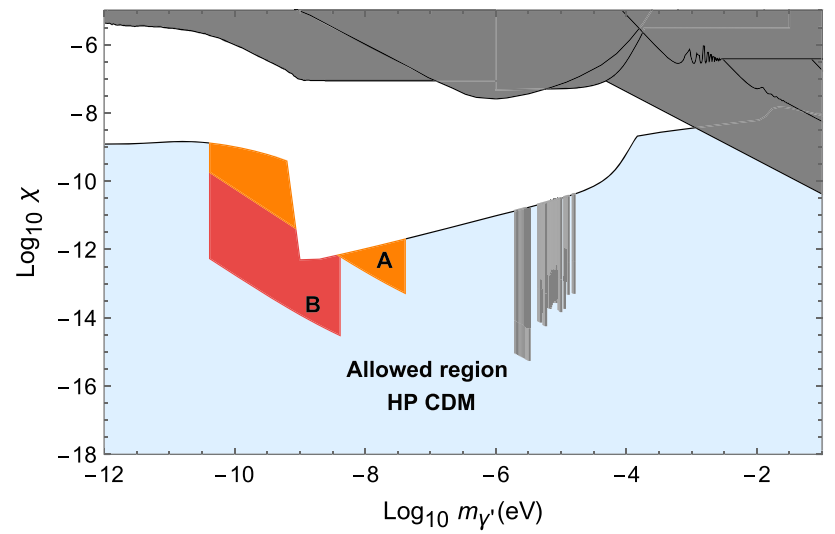

Fig. 2 Parameter space scanned by the LC-circuit proposed in [20] for hidden-photon cold dark matter (see the text for details)

in [20], leading to a reduced coherence time. The latter translates into a different magnetometer's sensitivity, now given by

$\delta B=10^{-16} \mathrm{~T}(\mathrm{~Hz})^{-1 / 2}\left(t_{\mathrm{c}} t\right)^{-1 / 4} ;$

see also [28] for a detailed discussion of the sensitivity scaling. If we consider the experiment to run parasitically to the search for axion DM, the measurement time is $t=10^{3} \mathrm{~s}$, and the coherence time is $t_{\mathrm{c}}=0.16 \mathrm{~s}(\mathrm{MHz} / v)$. In order to compare with [20] we have chosen a signal-to-noise ratio of 5 . In Fig. 2 we show the parameter space that could be scanned by the LC circuit proposed in [20] for hidden-photon cold dark matter. The light blue area corresponds to the allowed parameter space of hidden-photon cold dark matter [12]. The orange region corresponds to the sensitivity of the experiment running with the ADMX magnet (A), $V_{m}=0.023 \mathrm{~m}^{3}$, while the red region assume the setup working with the CMS magnet (B), $V_{m}=29.25 \mathrm{~m}^{3}$ (for details of these two magnets see [20]). Gray areas correspond to previously excluded regions. We have considered scenario (ii) and used $\kappa=0.5$. We emphasize again that the HP setup could in principle profit from the fact that is not necessary to magnetize the volume. However, we stick to the estimates above, because cryogenics and shielding at such values are demanding by themselves. This then sets the lower frequency for our scans, whereas we take the high-frequency cutoff through stray capacitance as in [20].

\section{Conclusions}

If cold dark matter is made of hidden photons, it can source electric and magnetic fields, since modifies Maxwell equations in a similar way as axions (and axion-like particles) do. We have pointed out that the proposal of Sikivie-SullivanTanner to search for axionic CDM with an LC-circuit could also be used to test the hidden-photons as dark matter candidate. The projected sensitivity of the experiment for hid- den photons can cover the unconstrained parameter space, as shown in Fig. 2.

We emphasize again that our proposal has the 'advantage' over [20] that no strong external magnet is needed, but rather just cryogenic volume and appropriate electronics.

We believe that this makes our proposal attractive to a larger group of experimentalists without access to strong magnets. Given the huge discovery potential for hiddenphoton cold dark matter, also a dedicated search that runs non-parasitically to the axionic equivalent constitutes a worthwhile fundamental physics experiment.

Acknowledgments We would like to thank Joerg Jaeckel and Pierre Sikivie for comments. This work was supported by FONDECYT/Chile grants and ACT1102 (P.A.), 1130020 (J.G.), 1140243 (F.M.) and Conicyt-21120890 (A. A.).

Note added in proof One week after the present paper was submitted to the arXiv, an extensive proposal for a DM search for hidden photons was put forward in [29]. Even though their detection technique of the hidden-photon DM condensate is different from the one considered here [20], they treated details of the important point that the setup needs to be shielded. We agree a shielding is needed and in fact is necessary to make it work in the magneto-quasistatic limit as implied in our calculation.

Open Access This article is distributed under the terms of the Creative Commons Attribution 4.0 International License (http://creativecomm ons.org/licenses/by/4.0/), which permits unrestricted use, distribution, and reproduction in any medium, provided you give appropriate credit to the original author(s) and the source, provide a link to the Creative Commons license, and indicate if changes were made.

Funded by $\mathrm{SCOAP}^{3}$.

\section{References}

1. G. Bertone, D. Hooper, J. Silk, Phys. Rep. 405, 279 (2005). arXiv:hep-ph/0404175

2. J. Redondo, A. Ringwald, Contemp. Phys. 52, 211 (2011). arXiv:1011.3741 [hep-ph]

3. R.D. Peccei, H.R. Quinn, Phys. Rev. Lett. 38, 1440 (1977)

4. S. Weinberg, Phys. Rev. Lett. 40, 223 (1978)

5. F. Wilczek, Phys. Rev. Lett. 40, 279 (1978)

6. J. Preskill, M.B. Wise, F. Wilczek, Phys. Lett. B 120, 127 (1983)

7. L.F. Abbott, P. Sikivie, Phys. Lett. B 120, 133 (1983)

8. M. Dine, W. Fischler, Phys. Lett. B 120, 137 (1983)

9. K. Baker, G. Cantatore, S.A. Cetin, M. Davenport, K. Desch, B. Döbrich, H. Gies, I.G. Irastorza et al., Ann. Phys. 525, A93 (2013). arXiv:1306.2841 [hep-ph]

10. B. Holdom, Phys. Lett. B 166, 196 (1986)

11. A.E. Nelson, J. Scholtz, Phys. Rev. D 84, 103501 (2011)

12. P. Arias, D. Cadamuro, M. Goodsell, J. Jaeckel, J. Redondo, A. Ringwald, JCAP 1206, 013 (2012)

13. A. Wagner et al., ADMX Collaboration. Phys. Rev. Lett. 105, 171801 (2010). arXiv:1007.3766 [hep-ex]

14. K. Ehret, M. Frede, S. Ghazaryan, M. Hildebrandt, E.A. Knabbe, D. Kracht, A. Lindner, J. List et al., Phys. Lett. B 689, 149 (2010). arXiv: 1004.1313 [hep-ex]

15. JINST 8, T09001 (2013) arXiv:1302.5647 [physics.ins-det]

16. M. Betz, F. Caspers, M. Gasior, M. Thumm, S. W. Rieger, Phys. Rev. D 88 (7), 075014 (2013) arXiv:1310.8098 [physics.ins-det]

17. I.G. Irastorza, F.T. Avignone, G. Cantatore, J.M. Carmona, S. Caspi, S.A. Cetin, F.E. Christensen, A. Dael et al., J. Phys. Conf. Ser. 460, 012002 (2013) 
18. D. Horns, J. Jaeckel, A. Lindner, A. Lobanov, J. Redondo, A. Ringwald, JCAP 1304, 016 (2013). arXiv:1212.2970

19. B. Döbrich, K. Daumiller, R. Engel, M. Kowalski, A. Lindner, J. Redondo, M. Roth, arXiv:1410.0200 [physics.ins-det]

20. P. Sikivie, N. Sullivan, D.B. Tanner, Phys. Rev. Lett. 112, 131301 (2014)

21. P.W. Graham, J. Mardon, S. Rajendran, Y. Zhao, Phys. Rev. D 90, 075017 (2014). arXiv:1407.4806 [hep-ph]

22. K.R. Dienes, C.F. Kolda, J. March-Russell, Nucl. Phys. B 492, 104 (1997)

23. S.A. Abel, M.D. Goodsell, J. Jaeckel, V.V. Khoze, A. Ringwald, JHEP 0807, 124 (2008)
24. M. Goodsell, J. Jaeckel, J. Redondo, A. Ringwald, JHEP 0911, 027 (2009)

25. M. Cicoli, M. Goodsell, J. Jaeckel, A. Ringwald, JHEP 1107, 114 (2011)

26. J. Hong, J. E. Kim, S. Nam, Y. Semertzidis, arXiv:1403.1576 [hep$\mathrm{ph}]$

27. M.S. Turner, Phys. Rev. D 33, 889 (1986)

28. D. Budker, P.W. Graham, M. Ledbetter, S. Rajendran, A. Sushkov, Phys. Rev. X 4, 021030 (2014). arXiv:1306.6089 [hep-ph]

29. S. Chaudhuri, P. W. Graham, K. Irwin, J. Mardon, S. Rajendran and Y. Zhao, arXiv:1411.7382 [hep-ph] 